\title{
Track Reconstruction and Muon Identification in the Muon Detector of the CBM Experiment at FAIR
}

\author{
Andrey Lebedev ${ }^{* a b}$, Claudia Höhne ${ }^{a}$, Ivan Kisel ${ }^{a}$, Anna Kiseleva ${ }^{a}$ and Gennady \\ Ososkov ${ }^{b}$ for the CBM collaboration ${ }^{\dagger}$ \\ ${ }^{a}$ GSI Helmholtzzentrum für Schwerionenforschung $\mathrm{GmbH}$ \\ 64291 Darmstadt, Germany \\ ${ }^{b}$ Joint Institute for Nuclear Research \\ 141980 Dubna, Russia \\ ${ }^{c}$ KIP, University of Heidelberg \\ 69117 Heidelberg, Germany \\ E-mail: andrey. lebedev@gsi.de
}

\begin{abstract}
The Compressed Baryonic Matter (CBM) experiment at the future FAIR accelerator at Darmstadt is being designed for a comprehensive measurement of hadron and lepton production in heavyion collisions from 8-45 AGeV beam energy. The setup consists of several detectors, including the silicon tracking system (STS) placed in a dipole magnet close to the target region, and the MUCH (MUon CHamber) detector. The MUCH detector is aimed for muon identification down to momenta of $1.5 \mathrm{GeV} / \mathrm{c}$. It consists of a sequence of absorber layers and detector stations. The concept for the MUCH detector and the status of the track reconstruction software are presented in this contribution. The reconstruction software is organized to be flexible with respect to feasibility studies of different physics channels and to optimization of the detector itself. The track reconstruction efficiency for muons embedded in central $\mathrm{Au}+\mathrm{Au}$ collisions at $25 \mathrm{AGeV}$ beam energy from the UrQMD model is at the level of $95 \%$. In these collisions, feasibility studies of low mass vector meson measurements in the dimuon channel result in $2 \%$ total reconstruction efficiency of the omega meson and a signal to background ratio of 0.09. Currently, ongoing work focuses on detector layout studies in order to optimize the detector setup while keeping a high performance.
\end{abstract}

XII Advanced Computing and Analysis Techniques in Physics Research November 3-7, 2008

Erice, Italy

\footnotetext{
* Speaker.

$\dagger$ This work was supported by the EU Integrated Infrastructure Initiative Hadron-Physics Project (I3HP) under Contract number RII3-CT-2004-506078.
} 

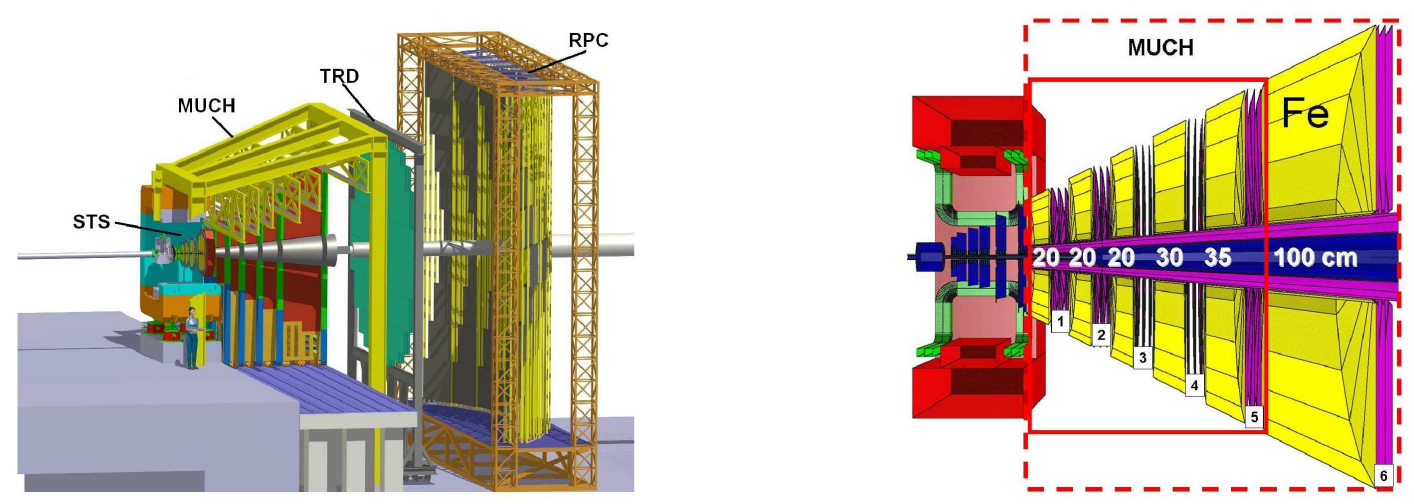

Figure 1: Sketch of the CBM detector [left] and a schematic view of the STS and muon detectors [right].

\section{Introduction of CBM}

An international accelerator facility FAIR (Facility for Antiproton and Ion Research) is being built at Darmstadt, Germany, for research with ion and antiproton beams. The heart of the new facility are two superconducting synchrotrons (SIS100 and SIS300) with a circumference of about 1,100 meters. Up to 5 various experimental halls will be served by the facility at a time. The accelerator will yield ion beams with highest beam intensity, e.g. $2 \times 10^{13} / \mathrm{s} 90 \mathrm{GeV}$ protons and $10^{9} \mathrm{Au}$ ions/s at $45 \mathrm{AGeV}$ beam energy. First experiments are planed for 2012, full operation is forseen for 2016.

The CBM experiment [1] is being designed to investigate high-energy nucleus-nucleus collisions at the future international FAIR project. The objective of high-energy heavy-ion collision experiments performed worldwide is to explore the phase diagram of matter governed by the laws of QCD. Of particular interest is the phase where a quark-gluon-plasma is formed. At very high beam energies - as provided by RHIC in Brookhaven and by LHC at CERN - matter is created at extremely high temperatures similar to the early universe. In heavy-ion collisions at FAIR beam energies (2-45 AGeV) nuclear matter is compressed to very high net-baryon densities as in core collapse supernovae, or in the interior of neutron stars. The CBM experiment offers the possibility to discover the most prominent landmarks of the QCD phase diagram expected to exist at high net baryon densities: the first order deconfinement phase transition and the critical endpoint. At these baryon densities also hadrons are expected to change their properties and chiral symmetry to be restored. Special focus is set on rare observables such as charm and dilepton production as those observables can give insight into the hot and dense medium created in heavy ion collisions.

The experimental task is a comprehensive measurement of hadron and lepton production in $p p$, $p A$ and $A A$ collisions from $8-45 A G e V$ beam energy. The experimental challenge is to select rare events in nucleus-nucleus collisions with charged particle multiplicities of about 1000 per central event at reaction rates of up to $10 \mathrm{MHz}$.

The CBM experimental setup for muon measurements comprises the following detector components (see Figure 1):

- Silicon Tracking System (STS) located inside a large acceptance dipole magnet for track and vertex reconstruction and momentum determination. 
- MUon CHamber absorber system (MUCH) for muon identification.

- Transition Radiation Detectors (TRD) for global track reconstruction.

- Resistive Plate Chambers (RPC) provide a time-of-flight measurement needed for hadron identification.

\section{The CBM muon identification system}

The CBM muon detector will be used for the detection of vector mesons $(\rho, \omega, \phi, J / \psi)$ via their decay into $\mu^{+} \mu^{-}$pairs. The muon detection system is located downstream of the STS. The standard muon identification by absorber technique is used. The actual design of the muon system consists of 5-6 hadron absorber layers made of iron of variable thickness, and of 3 tracking detectors in between each of the gaps. Currently, the detectors are foreseen to be made with GEM technology with a minimum pad size of $1.4 \times 2.8 \mathrm{~cm}^{2}$ (see Figure 1). For the measurement of muons from low-mass vector mesons $(\rho, \omega, \phi)$, the total iron absorber thickness is $125 \mathrm{~cm}(7.5$ $\lambda_{I}$ ), whereas for muons from charmonia, $1 \mathrm{~m}$ of iron is added (total thickness of $13.4 \lambda_{I}$ ).

\section{Track reconstruction}

The main track reconstruction blocks are track propagation, finder, fitter and selection.

The standard Kalman filter technique proposed in [2] has been used for the estimation of track parameters.

\subsection{Track propagation}

The track propagation algorithm calculates the average trajectory and corresponding errors. During the propagation three physics processes are taken into account: 1) energy loss; 2) multiple Coulomb scattering; 3 ) magnetic field. The main components of the track propagator are the track extrapolator, the material effects calculator and the geometry navigator. The track propagator manages these three blocks and performs the transport of the track.

The extrapolation part relates to the geometrical extrapolation, governed by the equations of motion. In case of the absence of a magnetic field a straight line model is used for the average trajectory and transport matrix calculation. In a magnetic field the equation of motion for a charged particle is solved with the $4^{\text {th }}$ order Runge-Kutta method with a parallel integration of the derivatives.

Material effects are taken into account by regularly updating the momentum due to energy loss and by adding process noise due to multiple scattering and energy loss to the track covariance matrix. Energy loss of particles traversing detector material occurs due to electromagnetic effects ionization (Bethe-Bloch formula), bremsstrahlung (Bethe-Heitler formula) and direct pair production. Multiple scattering is a random process, therefore it influences only the covariant matrix. A Gaussian approximation (Highland formula) [5] has been used to estimate the projected scattering angle.

The geometry navigator searches for intersection points with the detector elements in a certain segment along a straight line. The implementation of the navigation is based on the ROOT geometry package.

Assume that we want to propagate a track from $Z_{0}$ to $Z$ (see Figure 2). First, the segment $\left[Z_{0}, Z\right]$ is 


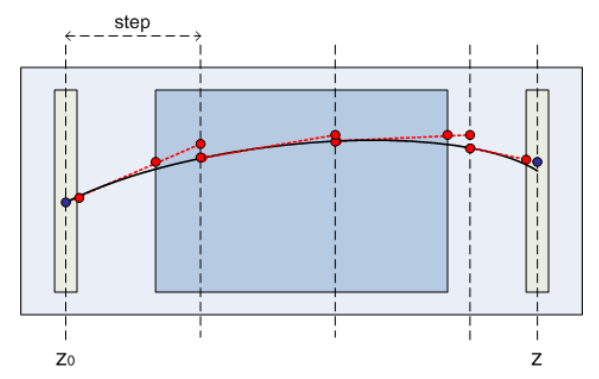

Figure 2: Sketch of the track propagation algorithm.

divided into several steps. In each of these steps the geometry navigator searches for intersections with the material along a straight line (marked with dots). The precise extrapolation is then done in a second step between the intersection points inside each step. Material effects are added at each intersection point. The straight line parameters as used in the navigation are updated after each step.

A detailed description of the developed track propagation algorithm can be found in [3].

\subsection{Track finding}

The track finding algorithm is based on track following and the Kalman filter. The algorithm tracks the particles and includes new hits one by one. It uses branching, where a branch is created for each hit that passed a test to be assigned to the track-segment. The algorithm accounts for missing hits in order to deal with detector inefficiencies. A flowchart of the algorithm is shown in Figure 3 (left). The algorithm consists of two main parts, i.e. track following and track selection. A sketch of the track following and creation of branches is shown in Figure 3 (right) for the example of two input tracks. The algorithm starts by extrapolating input tracks taken from the STS to the first station. The searching region is calculated as $\pm 3 \sigma$ around the predicted position assuming a Gaussian distribution of errors. Hits which are in this searching region are assigned to the track. For each of the hits and a possibly missing hit a separate branch is created. The parameters of each track-branch are updated with the Kalman filter. This procedure is repeated until the last station is reached. In the example shown, there is one hit in the searching region of track 1 on the $1^{\text {st }}$ station. As a result two branches are created - the first one for the hit, the second one for a possibly missing hit. Finally, for track 1 five branches have been created, two of them will be used for the further track selection; for the track 2 two branches have been created, one of them is good. Currently one missing hit is allowed for at maximum, branches with more are rejected from the sample.

\subsection{Track selection}

The aim of the track selection algorithm is to remove clone and ghost tracks and to keep good ones. Clone tracks are tracks that consist of a similar set of hits, ghost tracks are tracks that consist of some random set of hits. The algorithm works in two stages. First, tracks are sorted by their quality, which is defined by the track length and $\chi^{2}$ (see Figure 4). Then a procedure which checkes all shared hits of the tracks is executed. It loops over all tracks starting with the highest quality track and collects used hits, for each new track the number of shared hits is checked. If there are too many shared hits the track is rejected. 

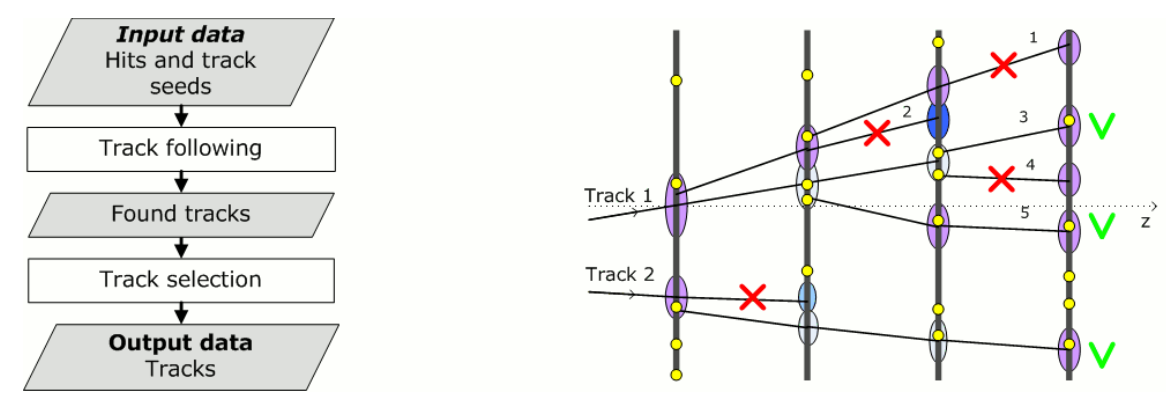

Figure 3: Flowchart [left] and sketch [right] of the track finding algorithm.

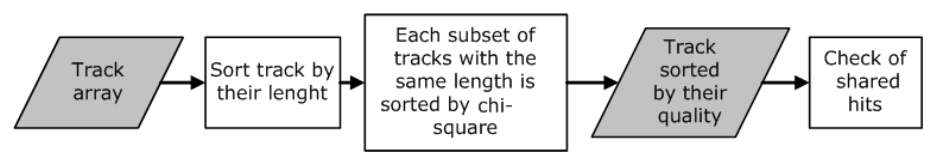

Figure 4: Track selection flowchart.

\begin{tabular}{|c|c|c|c|c|c|c|c|c|c|c|}
\hline & $x$ & $x_{G}$ & $y$ & $y_{G}$ & $t_{x}$ & $t_{x G}$ & $t_{y}$ & $t_{y G}$ & $q / p$ & $q / p_{G}$ \\
\hline residual & 3.39 & 3.39 & 3.43 & 3.42 & 3.26 & 3.26 & 3.26 & 3.26 & 1.23 & 1.06 \\
\hline pull & 1.09 & 1.13 & 1.09 & 1.13 & 1.10 & 1.08 & 1.10 & 1.09 & 1.49 & 0.74 \\
\hline
\end{tabular}

Table 1: Sigma values for residuals and pulls for a Gaussian fit to the corresponding distributions, values of the developed tracking routine are compared with those from GEANE (marked with G).

\section{Performance of the track reconstruction}

\subsection{Performance of the track propagation}

The developed track propagation algorithm was tested and compared with the GEANE track propagation package which is available in the CBMROOT framework within a $\mathrm{C}++$ interface [4]. The simulation was done for muons originating from the target with a momentum range of 1-10 $\mathrm{GeV} / \mathrm{c}$ and an iron absorber length of $125 \mathrm{~cm}$. Residual and pull distributions were calculated for the last MUCH station, results are presented in Table 1. The simulation shows a good agreement between the developed algorithm and GEANE.

\subsection{Performance of the track finder}

Central $\mathrm{Au}-\mathrm{Au}$ collisions at $25 \mathrm{AGeV}$ from the UrQMD model [6] were used to simulate the background, additionally 5 primary $\mu^{+}$and 5 primary $\mu^{-}$were embedded in each event to calculate the track reconstruction efficiency for primary muons. GEANT3 was used for transport. The efficiency was calculated for tracks which passed through the whole muon detector. The tracking performance is presented in Table 2 and in Figure 5.

\section{Feasibility studies using muon identification}

Vector mesons were simulated with the PLUTO generator [7] assuming an isotropic thermal source with a temperature of $130 \mathrm{MeV}$. Multiplicities for central $\mathrm{Au}+\mathrm{Au}$ collisions at $25 \mathrm{AGeV}$ 


\begin{tabular}{|c|c|c|}
\hline & $125 \mathrm{~cm} \mathrm{Fe}$ & $225 \mathrm{~cm} \mathrm{Fe}$ \\
\hline all & $96.2 \%$ & $97.5 \%$ \\
\hline muons & $96.8 \%$ & $97.5 \%$ \\
\hline ghosts & $1.5 \%$ & $0.2 \%$ \\
\hline
\end{tabular}

Table 2: Track finding efficiency.
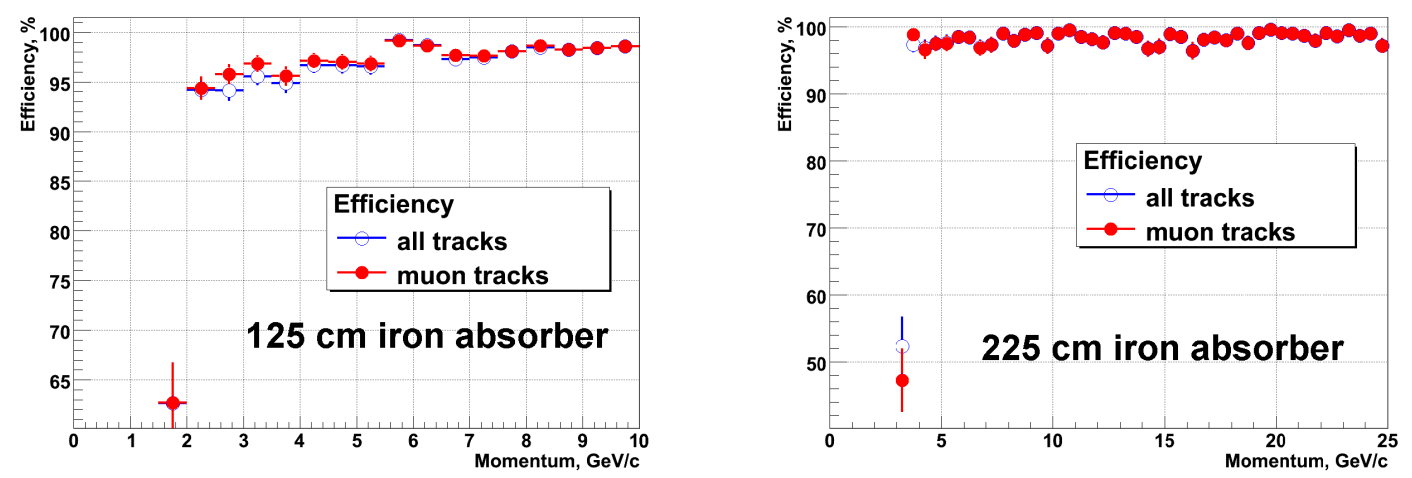

Figure 5: Track finding efficiency in dependence on momentum for $125 \mathrm{~cm}$ of iron [left] and $225 \mathrm{~cm}$ of iron [right]. (6\% statistical fluctuations are hidden by the chosen Binomial error calculation for these plots)

beam energy were taken from the HSD transport code [8]. The background was calculated with the UrQMD event generator. Both signal and background tracks were transported through the detector setup using the transport code GEANT3 within the CBMROOT simulation framework. Full track reconstruction in the STS and the muon system was done. Muon identification for low mass vector mesons require a track to pass through $125 \mathrm{~cm}$ of iron, for charmonium because of its higher momentum $225 \mathrm{~cm}$ of iron. Primary vertex cut and track $\chi^{2}$ cuts on the reconstructed tracks are made in order to suppress the background. After these analysis cuts about 0.25 background tracks per event are reconstructed on average for $\mathrm{Au}+\mathrm{Au}$ collisions at $25 \mathrm{AGeV}$ behind an iron absorber of $125 \mathrm{~cm}$ thickness. They consist of muons (52\%), kaons (30\%), pions (8\%), protons ( $3 \%$ ), and ghost tracks $(7 \%)$. About $80 \%$ of the muons originate from weak meson decays inside the absorber system, the rest from decays in front of the absorbers.

The resulting invariant mass spectra are shown in Figure 6 for the low-mass vector meson region (left) and the charmonium mass region (right). Efficiencies for vector meson detection and the signal-to-background ratio, calculated in a $\pm 2 \sigma$ window around the signal peaks are presented in Table 3. A lower transverse momentum cutoff at $1 \mathrm{GeV} / \mathrm{c}$ was applied for tracks in the charmonium region.

\section{Summary and outlook}

The proposed detector layout of alternating absorber-detector layers for muon identification in CBM is studied. A track reconstruction algorithm has been developed. It was succesfully demonstrated that tracking through the absorber is feasible with such a layout in a high track density environment: 97\% tracking efficiency for muons passing the absorber, a low ghost rate, few clone tracks and few track mismatches are achieved. 0.25 background tracks are reconstructed per cen- 

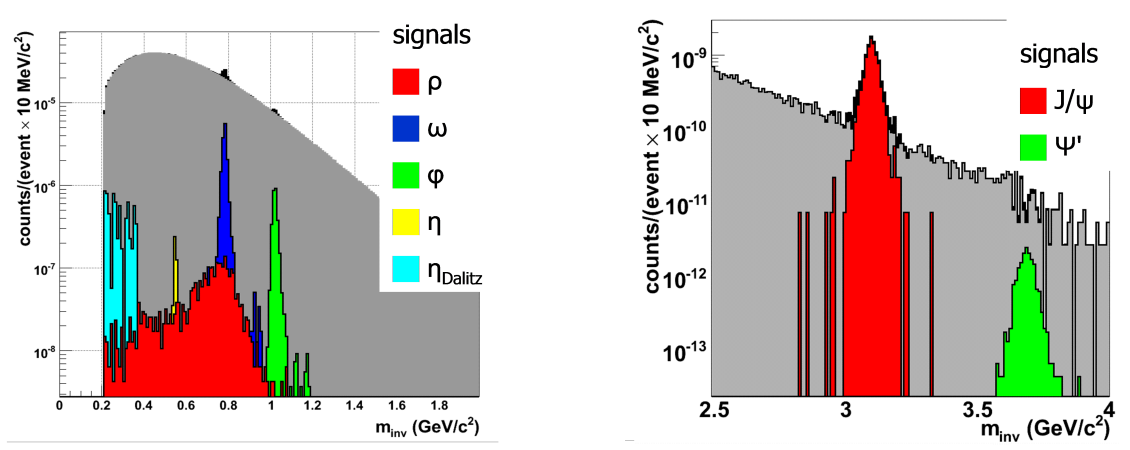

Figure 6: Muon pair invariant mass spectra. Left: low-mass region. Right: charmonium mass region.

\begin{tabular}{|c|c|c|c|}
\hline & Signal-to-Background (S/B) ratio & Efficiency (\%) & Mass resolution $(\mathrm{MeV} / \mathrm{c})$ \\
\hline$\rho$ & 0.001 & 2.7 & - \\
\hline$\omega$ & 0.08 & 3.7 & 10 \\
\hline$\phi$ & 0.03 & 6 & 12 \\
\hline$J / \psi$ & 7 & 16 & 24 \\
\hline$\Psi^{\prime}$ & 0.09 & 19 & 28 \\
\hline
\end{tabular}

Table 3: Reconstruction efficiencies, signal-to-background ratios and mass resolutions for vector mesons in central $\mathrm{Au}+\mathrm{Au}$ collisions at $25 \mathrm{AGeV}$. For the $\rho$ meason a mass range of $0.2-0.9 \mathrm{GeV} / \mathrm{c}^{2}$ was choosen for the $\mathrm{S} / \mathrm{B}$ ration.

tral $\mathrm{Au}+\mathrm{Au}$ collision at $25 \mathrm{AGeV}$ beam energy after $125 \mathrm{~cm}$ iron absorber; the main background are muons from $\pi$ and $\mathrm{K}$ decay. Physics feasibility studies on $J / \psi$ and low-mass vector meson reconstruction are promising.

\section{References}

[1] CBM Collaboration, Compressed Baryonic Matter Experiment. Technical Status Report, GSI Darmstadt (2005)

[2] R.Fruhwirth, Application of Kalman filtering to track and vertex fitting, Nucl. Instrum. Meth. A262 (1987) 444-450

[3] A.Lebedev, G.Ososkov, LIT Track Propagation for CBM, CBM note (2008) http://www.gsi.de/documents/DOC-2008-Dec-182-1.pdf

[4] A.Fontana et al., Track following in dense media and inhomogeneous magnetic fields, PANDA Report PV/01-07

[5] C. Amsler et al., The Review of Particle Physics, Physics Letters B667, 1 (2008)

[6] S.A.Bass et al., Microscopic Models for Ultrarelativistic Heavy Ion Collisions, Prog. Part. Nucl. Phys. 41 (1998) 225-370

[7] PLUTO generator http://www-hades.gsi.de/computing/pluto

[8] W.Cassing, E.L.Bratkovskaya and A.Sibirtsev, Nucl. Phys. A 691 (2001) 753 